\title{
Pressão arterial elevada em adolescentes de alto nível econômico
}

\author{
High blood pressure in adolescents of high economic status
}

\section{Diego Giulliano D. Christofaro¹, Juliano Casonatto ${ }^{2}$, Rômulo Araújo Fernandes ${ }^{3}$, Felipe Fossati Reichert', Mathias Roberto Lock ${ }^{4}$, Débora Alves Guariglia ${ }^{5}$, Milene Granja Sacomanni², Arli Ramos de Oliveira ${ }^{4}$}

\section{RESUMO}

Objetivo: Verificar a prevalência e os fatores associados à ocorrência da pressão arterial elevada em adolescentes de alto nível econômico.

Métodos: Foram investigados 233 alunos (113 meninos e 120 meninas) matriculados entre a quinta e a oitava série do ensino fundamental de escolas privadas de Londrina, Paraná. Foram coletadas informações sobre classificação econômica e os hábitos ligados ao consumo alimentar, por meio de questionários. A massa corporal foi avaliada por balança digital da marca Plenna e a altura por estadiômetro de madeira com precisão de $0,1 \mathrm{~cm}$. A pressão arterial foi aferida por aparelho oscilométrico automático. Para a análise estatística, utilizou-se o teste do qui-quadrado ou o teste $t$ para avaliar a associação entre a pressão arterial e os fatores de risco. A regressão de Poisson indicou a magnitude dessas associações.

Resultados: A prevalência da pressão arterial elevada foi de $12,4 \%$ entre os estudantes. Foram verificadas associações entre a pressão arterial elevada e as variáveis: sexo e estado nutricional. Posteriormente, no modelo ajustado, a regressão de Poisson somente indicou significância entre a associação da pressão arterial elevada e o estado nutricional.

Conclusões: Observou-se significativa prevalência de pressão arterial elevada em adolescentes de alto nível econômico e os valores elevados de pressão arterial se associaram ao excesso de peso corporal.
Palavras-chave: adolescente; prevalência; pressão arterial; obesidade.

\section{ABSTRACT}

Objective: To verify the association between elevated blood pressure and related risks factors on adolescents of high economic level.

Methods: The sample included 233 schoolchildren (113 boys and 120 girls) on the fifth to the eighth grades of private elementary schools from Londrina, Paraná, Brazil. The subjects' economic level and food intake were evaluated by a questionnaire. The body mass was assessed with a Plenna digital scale; the height was measured with a wooden stadiometer (precision: $0.1 \mathrm{~cm}$ ); and the blood pressure, with an oscillometric device. Association between elevated blood pressure and different risk factors were evaluated by the t-test, chi-square test and by Poisson's regression with robust variance.

Results: Elevated blood pressure was present in $12.4 \%$ of the students and it was associated with gender and nutritional status. Poisson's regression adjusted model suggested that only nutritional status was independently associated with elevated blood pressure.

Conclusions: A high rate of elevated blood pressure was found in adolescents from high economic level, and overweight was associated with this condition.

Key-words: adolescent; prevalence; blood pressure; obesity.
Instituição: Universidade Estadual de Londrina (UEL), Londrina, PR, Brasil

'Doutorando em Saúde Coletiva pela UEL; docente do curso de Educação Física da Universidade Estadual Paulista (Unesp), Presidente Prudente, SP, Brasil

${ }^{2}$ Mestrando em Educação Física pela UEL, Londrina, PR, Brasil

${ }^{3}$ Doutorando em Ciências da Motricidade pela Unesp, Rio Claro, SP, Brasil

${ }^{4}$ Docente do curso de Educação Física da UEL, Londrina, PR, Brasil

${ }_{5}^{5}$ Mestre em Educação Física pela UEL, Londrina, PR, Brasil
Endereço para correspondência:

Juliano Casonatto

Avenida São João, 1.329 - Residencial Metropolitan Plaza, G-72

CEP 86039-270 - Londrina/PR

E-mail: julianoc@sercomtel.com.br

Conflito de interesse: nada a declarar

Recebido em: 25/2/2009

Aprovado em: 4/7/2009 


\section{Introdução}

Atualmente, alguns importantes fatores de risco para o desenvolvimento de doenças cardíacas, como a hipertensão arterial, têm sido identificados em idades relativamente precoces ${ }^{(1)}$. Além disso, estudos longitudinais têm demonstrado que tais fatores de risco desenvolvidos durante a infância e a adolescência tendem a se manter na vida adulta ${ }^{(2-4)}$.

Dessa forma, a busca/definição por fatores associados à hipertensão arterial em populações pediátricas é o foco de muitos pesquisadores. Nesse sentido, entre populações jovens, o aumento significativo na ocorrência da obesidade no mundo tem sido motivo de muita preocupação para diferentes profissionais da área da saúde, uma vez que esta condição associa-se ao surgimento de disfunções de caráter crônico-degenerativo, como a hipertensão $\operatorname{arterial}^{(5)}$. Nessa direção, embora tenha havido um aumento na taxa de obesidade nas classes econômicas mais baixas; no Brasil, a maior prevalência ainda ocorre nos estratos econômicos mais elevados $^{(6,7)}$, tornando, por sua vez, este estrato da população um grupo de aparente maior risco ao desenvolvimento de desfechos associados à obesidade.

Além disso, o consumo alimentar é outro fator que pode estar independentemente associado à pressão arterial. Em adultos, por exemplo, o consumo excessivo de sal e a elevada ingestão de cafeína parecem colaborar para a elevação da pressão arterial. Contudo, em adolescentes, a relação entre hábitos alimentares e níveis pressóricos ainda não está esclarecida, uma vez que alguns estudos identificaram tal relação ${ }^{(8)}$ e outros, não ${ }^{(9,10)}$.

Nesse sentido, o objetivo do presente estudo foi verificar a prevalência de pressão arterial elevada (PAE) em adolescentes de alto nível econômico da cidade de Londrina, Paraná, bem como explorar sua associação com alguns fatores de risco.

\section{Métodos}

Estudo descritivo/analítico de delineamento transversal, realizado na cidade de Londrina, Paraná. A coleta dos dados ocorreu no ano de 2008 entre os meses de março e maio. A amostra analisada foi composta por alunos da quinta a oitava série do ensino fundamental, com idade entre dez e 15 anos. A idade centesimal dos estudantes foi determinada pela diferença entre a data de nascimento e o dia da avaliação. Os critérios para a inclusão dos jovens no estudo foram baseados em três informações: 1) deveriam estar devidamente matriculados em uma das instituições de ensino em questão; 2) não apresentar nenhum tipo de doença metabólica diagnosticada ou utilizar medicamentos para o controle da pressão arterial ou estar em processo de gestação; 3) retornar com o termo de consentimento livre esclarecido devidamente assinado.

Segundo dados da Secretaria Municipal de Ensino, o município de Londrina possui 8.398 alunos matriculados em instituições particulares de ensino (dados de 2007). Dessa forma, a cidade foi dividida em seis regiões: norte, sul, leste, oeste, central e anel periférico. Em cada região foi sorteada uma escola e, de cada uma, avaliou-se aleatoriamente um número de turmas que atingisse a proporcionalidade de estudantes matriculados nas respectivas regiões.

Para o cálculo do tamanho da amostra foram empregados os procedimentos sugeridos por Luiz e Magnanini ${ }^{(11)}$, sendo adotado nível de confiança de $95 \%$ e erro tolerável de 5\%. A prevalência estimada de hipertensão arterial foi de $10 \%$, baseada em levantamento anterior ${ }^{(12)}$. Dada à dificuldade de conseguir uma relação nominal dos alunos e principalmente a questão operacional que este método implicaria, optou-se pela realização da coleta considerando-se a turma por completo (conglomerados), sendo realizada uma correção amostral (efeito do design - deff) de 1,5 , estimando um tamanho mínimo da amostra de 204 escolares. A esta estimativa acrescentou-se mais 20\% referentes a possíveis perdas amostrais. Em conformidade com o plano amostral, 245 estudantes participaram do estudo.

Dos 245 escolares, aproximadamente $5 \%(\mathrm{n}=12)$ faltaram no dia da avaliação ou se recusaram a realizar as medidas. A amostra final foi de 233 (120 meninos e 113 meninas), mantendo-se o poder estatístico inicial e a representatividade da população. $\mathrm{O}$ estudo seguiu as diretrizes e normas que regulamentam a pesquisa com seres humanos, sendo informado a todos os participantes e responsáveis os métodos, propósitos e benefícios do estudo. Anteriormente ao início de qualquer avaliação, todos os participantes e seus respectivos responsáveis assinaram um termo de consentimento livre e esclarecido. A pesquisa foi aprovada pelo Comitê de Ética da Universidade Estadual de Londrina.

Após a realização da pesquisa, os diretores e pais de alunos das escolas participantes do estudo receberam os resultados das avaliações dos adolescentes (pressão arterial, índice de massa corporal e hábitos alimentares). Para os adolescentes com valores elevados de pressão arterial e/ou excesso de peso corporal, foi recomendado aos pais que os conduzissem a uma consulta médica. Quanto aos adolescentes com alimentação imprópria, sugeriu-se uma consulta a um nutricionista. 
O Critério de Classificação Econômica do Brasil, estabelecido pela Associação Brasileira de Antropologia e Associação Nacional de Empresas de Pesquisa ${ }^{(13)}$, foi empregado para determinação do nível econômico. O instrumento foi preenchido pelo próprio escolar em sala de aula com o auxílio de um avaliador. Os indivíduos foram classificados em nível alto (A e B) e baixo (C, D e E).

Os hábitos relacionados à ingestão alimentar foram determinados por meio de um instrumento desenvolvido especificamente para o estudo. Ele foi composto por seis perguntas, no qual os avaliados responderam em quantos dias (de zero a sete dias) na semana consumiram determinados tipos de alimentos (salgadinhos, frutas, carnes vermelhas/frituras, salgadinhos tipo chips, refrigerantes e verduras/legumes). O consumo de "salgadinhos" do tipo chips foi utilizado como indicador do consumo de sal, visto que o consumo desse tipo de alimento parece ser mais comum na adolescência.

$\mathrm{O}$ indicador do consumo de gorduras foi determinado pela ingestão de carne vermelha, salgados fritos e doces. Para indicar o consumo de cafeína, foi avaliada a ingestão semanal de refrigerantes do tipo "cola”. Avaliou-se também o consumo semanal de frutas e legumes. Foi considerado alto consumo quando os alimentos eram consumidos com frequência superior a três vezes na semana e, baixo consumo, se ingeridos com assiduidade igual ou inferior a três vezes na semana.

Após 15 dias da primeira administração do questionário alimentar, determinou-se que aproximadamente $15 \%$ da amostra ( $n=42$, escolhidos aleatoriamente) deveria preenchêlo novamente para verificar sua reprodutibilidade. Os resultados foram avaliados pelo índice Kappa (k), que verificou elevada concordância entre os dois momentos de aplicação $(k=0,83)$.

A massa corporal foi aferida com a utilização de uma balança digital da marca Plenna, com precisão de $0,1 \mathrm{~kg}$ e capacidade máxima de $150 \mathrm{~kg}$. Os avaliados permaneceram descalços, posicionados em pé, no centro da plataforma da balança e vestindo roupas leves. A estatura foi aferida com a utilização de um estadiômetro fixo de madeira com precisão de $0,1 \mathrm{~cm}$. Os avaliados permaneceram na posição ortostática, descalços, voltados de costas para a superfície vertical do aparelho e a cabeça posicionada no plano de Frankfurt, os membros superiores relaxados ao lado do tronco, com as palmas das mãos voltadas para as coxas, os calcanhares unidos, tocando a parte vertical do estadiômetro e as bordas mediais afastadas.

O índice de massa corpórea (IMC) foi calculado pela divisão da massa corporal pelo valor da estatura elevado ao quadrado $\left(\mathrm{kg} / \mathrm{m}^{2}\right)$, sendo utilizados os valores críticos específicos para sexo e idade, propostos por Cole et a ${ }^{(14)}$ para indicar a presença de sobrepeso e obesidade.

Para a aferição da pressão arterial foi utilizado um aparelho oscilométrico automático (Omron-HEM-742), previamente validado ${ }^{(15)}$, com manguitos específicos para crianças e adolescentes ${ }^{(16)}$. A pressão arterial foi aferida no braço direito após um período de repouso (sentado) de, no mínimo, cinco minutos. Subsequentemente à primeira avaliação dos sujeitos, padronizou-se um intervalo de dois minutos para uma segunda avaliação. Os valores de pressão arterial sistólica e diastólica foram estimados pela média das duas avaliações.

Para determinar pressão arterial elevada (PAE) foram adotados os pontos de corte recomendados pela literatura ${ }^{(17)}$, sendo considerados portadores de PAE todos os indivíduos que apresentaram valores de pressão arterial sistólica e/ou diastólica acima do percentil 95 para sua respectiva idade e estatura.

Para verificar eventuais diferenças entre as variáveis contínuas em relação ao gênero (idade, IMC, pressão arterial sistólica e diastólica), foi utilizado o teste $t$ de Student para amostras independentes. No modelo univariado, para a análise das associações entre a PAE e os fatores de risco investigados, empregou-se o teste do qui-quadrado. Com o objetivo de analisar a magnitude dessas associações, empregou-se a regressão de Poisson com ajuste robusto de variância. No modelo ajustado da regressão de Poisson, todas as variáveis independentes foram inseridas simultaneamente. Valores de $p$ inferiores a $5 \%$ foram considerados estatisticamente significantes. A análise estatística foi feita com o software STATA 8.0.

\section{Resultados}

Todos os 233 alunos foram classificados em nível econômico A e B. As características gerais dos adolescentes são apresentadas na Tabela 1. Entre os sexos, não houve diferenças para a idade e para a pressão arterial diastólica. Entretanto, as médias de pressão arterial sistólica e o IMC foram superiores entre os meninos, quando comparados às meninas.

A prevalência da PAE encontrada no estudo foi de 12,4\% (IC95\%=8,3-17,8\%) e o excesso de peso esteve presente em 27,1\% (IC95\%=21,4-33,2\%) dos estudantes. A Tabela 2 apresenta as associações brutas entre PAE e os fatores de risco analisados no estudo. A prevalência de PAE foi similar entre os sexos e mais frequente entre aqueles com sobrepeso e/ou obesidade. Houve associação entre PAE e o consumo de carne vermelha. 
Tabela 1 - Características gerais da amostra analisada

\begin{tabular}{lccccc}
\hline & \multicolumn{2}{c}{ Meninos $(\mathbf{n = 1 2 0})$} & \multicolumn{2}{c}{ Meninas $\mathbf{( n = 1 1 3 )}$} & \multirow{2}{*}{ Teste $\boldsymbol{t}$} \\
\cline { 2 - 4 } & \multicolumn{1}{c}{ Média } & DP & Média & DP & \\
\hline Idade $($ anos $)$ & 12,2 & 1,2 & 12,3 & 1,2 & 0,599 \\
IMC $\left(\mathrm{kg} / \mathrm{m}^{2}\right)$ & 20,4 & 3,9 & 19,4 & 2,7 & 0,031 \\
PAS $(\mathrm{mmHg})$ & 117,6 & 10,8 & 113,1 & 11,3 & 0,002 \\
PAD $(\mathrm{mmHg})$ & 64,4 & 8,2 & 65,3 & 7,9 & 0,413 \\
\hline
\end{tabular}

DP: desvio padrão; IMC: índice de massa corporal; PAS: pressão arterial sistólica; PAD: pressão arterial diastólica.

Tabela 2 - Ocorrência de pressão arterial elevada e fatores associados entre escolares da rede privada de ensino

\begin{tabular}{|c|c|c|c|}
\hline & $\begin{array}{c}\mathrm{PA} \geq \mathrm{P} 95 \\
\mathrm{n}(\%)\end{array}$ & RP (IC95\%) & Valor de $p$ \\
\hline Sexo & & & 0,534 \\
\hline Meninas & $17(14,2)$ & 1 & \\
\hline Meninos & $12(10,6)$ & $0,74(0,37-150)$ & \\
\hline Estado nutricional & & & 0,001 \\
\hline Eutrófico & $10(5,9)$ & 1 & \\
\hline Sobrepeso/obeso & $19(30,2)$ & $5,12(2,51-10,43)$ & \\
\hline Carne vermelha & & & 0,03 \\
\hline Normal & $27(15,4)$ & 1 & \\
\hline Elevado & $2(3,4)$ & $0,22(0,05-0,91)$ & \\
\hline Frutas & & & 1 \\
\hline Normal & $11(12,8)$ & 1 & \\
\hline Reduzido & $18(12,2)$ & $0,95(0,47-1,93)$ & \\
\hline Verduras & & & 0,939 \\
\hline Normal & $13(11,8)$ & 1 & \\
\hline Reduzido & $16(13,1)$ & $1,10(0,55-2,18)$ & \\
\hline Chips & & & 0,055 \\
\hline Normal & $28(15,1)$ & 1 & \\
\hline Elevado & $1(2,2)$ & $0,14(0,02-1,04)$ & \\
\hline Doces & & & 0,784 \\
\hline Normal & $16(13,4)$ & 1 & \\
\hline Elevado & $13(11,4)$ & $0,84(0,42-1,68)$ & \\
\hline Refrigerantes & & & 0,278 \\
\hline Normal & $20(14,8)$ & 1 & \\
\hline Elevado & $9(9,2)$ & $0,61(0,29-1,30)$ & \\
\hline
\end{tabular}

PA $\geq P 95$ : valor de PAS e ou PAD igual ou superior ao percentil 95; RP: razão de prevalência; IC95\%: intervalo de confiança de $95 \%$.

Tabela 3 - Modelo ajustado para a ocorrência de pressão arterial elevada e fatores associados entre escolares da rede privada de ensino

\begin{tabular}{llcc}
\hline & Referência & PA $\geq \mathbf{P 9 5}$ & \multirow{2}{*}{ Valor de $\boldsymbol{p}$} \\
\cline { 3 - 3 } & Meninos & $0,94(0,49-1,80)$ & 0,868 \\
Estado nutricional & Sobrepeso/obeso & $5,58(2,72-11,45)$ & 0,001 \\
Carne vermelha & Consumo elevado & $0,45(0,06-3,11)$ & 0,422 \\
Frutas & Consumo reduzido & $0,76(0,39-1,45)$ & 0,414 \\
Verduras & Consumo reduzido & $0,90(0,44-1,81)$ & 0,769 \\
Chips & Consumo elevado & $0,34(0,02-5,24)$ & 0,444 \\
Doces & Consumo elevado & $1,54(0,84-2,85)$ & 0,16 \\
Refrigerantes & Consumo elevado & $0,74(0,36-1,51)$ & 0,414 \\
\hline
\end{tabular}

PA $\geq P 95$ : valor de PAS e ou PAD igual ou superior ao percentil 95; RP: razão de prevalência; IC95\%: intervalo de confiança de $95 \%$. 
$\mathrm{Na}$ Tabela 3 são apresentados os valores de razão de prevalência ajustados entre as variáveis analisadas e a presença de PAE. O excesso de peso manteve-se associado a valores de PAE em repouso. Quando analisadas as associações estratificadas por sexo, novamente, apenas o excesso de peso manteve-se associado à PAE (Tabela 4).

\section{Discussão}

A prevalência de PAE na amostra analisada foi de 12,4\%, tais valores são próximos aos $15 \%$ encontrados por Nogueira et $a l^{(18)} \mathrm{em}$ escolares da cidade de Santos, São Paulo; porém, são superiores aos 9,4\% encontrados por Moura et al ${ }^{(12)}$ na cidade de Maceió, Alagoas e aos $8 \%$ indicados pela Sociedade Brasileira de Cardiologia ${ }^{(16)}$, como ocorrência média do evento na população pediátrica brasileira. A presente investigação envolveu apenas alunos da rede privada de ensino e este fator pode ter sido determinante na elevada ocorrência de PAE observada, uma vez que alunos da rede privada de ensino aparentemente possuem maior prevalência de fatores de risco à saúde $\mathrm{d}^{(19-21)}$.

Verificou-se, ainda, que apenas a variável estado nutricional manteve-se associada na análise multivariável. Este achado está de acordo com outros estudos nacionais e internacionais que examinaram a relação entre obesidade e pressão arterial, tanto em adultos ${ }^{(22)}$ quanto na população jovem ${ }^{(5)}$, indicando que a obesidade exerce impacto significativo sobre a pressão arterial.
No Brasil, a ocorrência de excesso de peso em crianças e adolescentes de escolas privadas parece ser superior àquela observada em estudantes da rede pública ${ }^{(19,20)}$. A ocorrência de excesso de peso na presente amostra foi de $27,1 \%$, similar ao observado por Nunes, Figueiroa e Alves ${ }^{(23)}$ em escolares de classe econômica alta de Campina Grande, Paraíba (31,4\%). Contudo, quando é estabelecida uma comparação com os resultados do mesmo estudo, porém, com o estrato de classe econômica baixa, os presentes achados são superiores; e superiores também aos dados da população brasileira na década de 1990, apresentados por Wang, Monteiro e Popkin ${ }^{(6)}$. Além disso, esta associação positiva entre classe econômica e excesso de peso também já foi observada em estudos anteriores realizados em território nacional por Fernandes et $a l^{(24)}$, em escolares de Presidente Prudente, São Paulo.

O presente estudo não identificou associação significativa entre hábito alimentar e PAE. Em adultos, o consumo satisfatório de frutas, verduras e legumes, atrelado à diminuição da ingestão de alimentos que detenham gorduras saturadas e sódio na sua composição, contribuem para reduzir os valores de pressão arterial em hipertensos ${ }^{(23)}$.

Porém quando a população estudada é composta por crianças e adolescentes, observa-se uma lacuna quanto à relação entre hábitos alimentares e PAE. Em outro estudo realizado também na cidade de Londrina, Guedes et al ${ }^{(8)}$ identificaram que os adolescentes que consumiam mais gorduras saturadas detinham maiores chances de ter hi-

Tabela 4 - Modelo ajustado para a ocorrência de pressão arterial elevada e fatores associados entre escolares de ambos os sexos da rede privada de ensino

\begin{tabular}{|c|c|c|c|c|}
\hline & \multicolumn{2}{|c|}{ Masculino com PA>P95 } & \multicolumn{2}{|c|}{ Feminino com PA>P95 } \\
\hline & RP (IC95\%) & Valor de $p$ & RP (IC95\%) & Valor de $p$ \\
\hline $\begin{array}{l}\text { Estado nutricional } \\
\text { Sobrepeso/obeso }\end{array}$ & $5,63(2,05-15,4)$ & 0,001 & $4,94(1,74-13,9)$ & 0,003 \\
\hline $\begin{array}{l}\text { Carne vermelha } \\
\text { Consumo elevado }\end{array}$ & $0,69(0,11-4,28)$ & 0,694 & $0,45(0,06-5,73)$ & 0,55 \\
\hline $\begin{array}{l}\text { Frutas } \\
\text { Consumo reduzido }\end{array}$ & $0,73(0,30-1,77)$ & 0,494 & $0,72(0,26-2,01)$ & 0,541 \\
\hline $\begin{array}{l}\text { Verduras } \\
\text { Consumo reduzido }\end{array}$ & $0,60(0,24-1,45)$ & 0,259 & $1,73(0,58-5,17)$ & 0,322 \\
\hline $\begin{array}{l}\text { "Chips" } \\
\text { Consumo elevado }\end{array}$ & $0,46(0,03-6,47)$ & 0,572 & $0,80(0,17-3,76)$ & 0,786 \\
\hline $\begin{array}{l}\text { Doces } \\
\text { Consumo elevado }\end{array}$ & $1,69(0,80-3,55)$ & 0,166 & $1,54(0,60-3,98)$ & 0,367 \\
\hline $\begin{array}{l}\text { Refrigerantes } \\
\text { Consumo elevado }\end{array}$ & $0,49(0,17-1,38)$ & 0,18 & $1,12(0,40-3,10)$ & 0,821 \\
\hline
\end{tabular}

PA $\geq P 95$ : valor de PAS e ou PAD igual ou superior ao percentil 95; RP: razão de prevalência; IC95\%: intervalo de confiança de $95 \%$. 
pertensão arterial, comparados aos seus pares com menor ingestão. Entretanto, quando as variáveis de confusão foram controladas por Pillegi et a $l^{(9)} \mathrm{em}$ um estudo com adolescentes italianos, não foi encontrada significância entre consumo alimentar e pressão arterial. Reddy et al $l^{(10)}$ também não encontraram associação significativa entre PAE e consumo de cafeína em meninas do continente africano. No entanto, vale destacar que o questionário do presente estudo forneceu apenas um indicativo quanto ao consumo semanal de gorduras saturadas, não permitindo análises adicionais. Além disso, recomenda-se que estudos futuros realizem a avaliação da quantidade de aldosterona a fim de averiguar possível influência de variável de confusão, uma vez que esse hormônio é responsável pelo balanço do sódio e potássio no sangue, o que consequentemente pode gerar aumento dos valores de pressão arterial.

\section{Referências bibliográficas}

1. Williams CL, Hayman LL, Daniels SR, Robinson TN, Steinberger J, Paridon S et al. Cardiovascular health in childhood: a statement for health professionals from the Committee on Atherosclerosis, Hypertension, and Obesity in the Young (AHOY) of the Council on Cardiovascular Disease in the Young, American Heart Association. Circulation 2002;106:143-60.

2. Lauer RM, Clarke WR. Childhood risk factors for high adult blood pressure: the Muscatine Study. Pediatrics 1989;84:633-41.

3. Freedman DS, Khan LK, Serdula MK, Dietz WH, Srinivasan SR, Berenson GS. The relation of childhood BMI to adult adiposity: the Bogalusa Heart Study. Pediatrics 2005;115:22-7.

4. Goran MI, Gower BA. Abdominal obesity and cardiovascular risk in children. Coron Artery Dis 1998;9:483-7.

5. Papandreou D, Rousso I, Makedou A, Arvanitidou M, Mavromichalis I. Association of blood pressure, obesity and serum homocysteine levels in healthy children. Acta Paediatr 2007;96:1819-23.

6. Wang $Y$, Monteiro C, Popkin BM. Trends of obesity and underweight in older children and adolescents in the United States, Brazil, China, and Russia. Am J Clin Nutr 2002;75:971-7.

7. da Veiga GV, da Cunha AS, Sichieri R. Trends in overweight among adolescents living in the poorest and richest regions of Brazil. Am J Public Health 2004;94:1544-8.

8. Guedes DP, Guedes JE, Barbosa DS, de Oliveira JA, Stanganelli LC. Cardiovascular risk factors in adolescents: biological and behavioral indicators. Arq Bras Cardiol 2006;86:439-50.

9. Pileggi C, Carbone V, Nobile CG, Pavia M. Blood pressure and related cardiovascular disease risk factors in 6-18 year-old students in Italy. J Paediatr Child Health 2005;41:347-52.

10. Reddy JG, Ebbert JO, Klesges LM, Enders FT, Klesges RC, Lanctot JQ et al. The relationship between caffeine and blood pressure in preadolescent African American girls. Ethn Dis 2008;18:283-8.

11. Luiz RR, Magnanini MM. The logic of sample size determination in epidemiological research. Cad Saude Coletiva 2000;8:9-28.

12. Moura AA, Silva MA, Ferraz MR, Rivera IR. Prevalence of high blood pressure in children and adolescents from the city of Maceió, Brazil. J Pediatr (Rio J) 2004;80:35-40.
Outra limitação a ser considerada é a possibilidade da causalidade reversa, já que a amostra pode ter modificado seus hábitos alimentares em um período próximo ao preenchimento do questionário, o que pode ter invertido as associações. Além disso, o modelo transversal impossibilita a inferência de relações de causalidade entre a variável desfecho e os diferentes fatores de risco analisados. Vale ressaltar que as medidas de pressão arterial acima do percentil 90 para a idade não foram confirmadas pelo método auscultatório, por outro lado, o número relativamente elevado de sujeitos que compõe o estudo reduz esse possível viés.

Pode-se concluir que a prevalência observada de PAE foi de 10,6\% em adolescentes de alto nível econômico do sexo masculino e $14,2 \%$ para o sexo feminino. Além disso, o estado nutricional desfavorável, como o sobrepeso e a obesidade, aparentemente estão associados à ocorrência de PAE na adolescência.
13. Instituto Brasileiro de Opinião Pública e Estatística [homepage on the Internet]. Levantamento sócio econômico-2000-IBOPE. [Cited 2003 Feb 2]. Available from: http://www.anep.org.br.

14. Cole TJ, Bellizzi MC, Flegal KM, Dietz WH. Establishing a standard definition for child overweight and obesity worldwide: international survey. Brit Med J 2000;320:1240-3.

15. Christofaro DG, Fernandes RA, Gerage AM, Alves MJ, Polito MD, Oliveira AR. Validação do monitor de medida de pressão arterial Omron HEM 742 em adolescentes. Arq Bras Cardiol 2009;92:10-5.

16. Sociedade Brasileira de Cardiologia [homepage on the Internet]. V Diretrizes Brasileiras de Hipertensão Arterial. [Cited 2009 Feb 20]. Available from: http://www. sbn.org.br/Diretrizes/N_Diretrizes_Brasileiras_de_Hipertensao_Arterial.pdf

17. National High Blood Pressure Education Program Working Group on High Blood Pressure in Children and Adolescents. The fourth report on the diagnosis, evaluation, and treatment of high blood pressure in children and adolescents. Pediatrics 2004;114:555-76.

18. Nogueira PCK, da Costa RF, Cunha JSN, Silvestrini L, Fisberg M. Pressão arterial elevada em escolares de Santos: relação com a obesidade. Rev Assoc Med Bras 2007;53:426-32.

19. da Costa RF, Cintra IP, Fisberg M. Prevalência de sobrepeso e obesidade em escolares da cidade de Santos, SP. Arq Bras Endocrinol Metab 2006;50:60-7.

20. Campos LA, Leite AJ, Almeida PC. Prevalence of overweight and obesity among adolescent students in the city of Fortaleza, Brazil. Rev Bras Saude Matern Infant 2007;7:183-90.

21. Scherr C, Magalhães CK, Malheiros W. Lipid profile analysis in school children. Arq Bras Cardiol 2007;89:65-70.

22. Adrogué HE, Sinaiko AR. Prevalence of hypertension in junior high schoolaged children: effect of new recommendations in the 1996 Updated Task Force Report. Am J Hypertens 2001;14:412-4.

23. Nunes MM, Figueiroa JN, Alves JG. Overweight, physical activity and foods habits in adolescents from different economic levels, Campina Grande (PB). Rev Assoc Med Bras 2007;53:130-4.

24. Fernandes RA, Casonatto J, Christofaro DGD, Ronque, ERV, Oliveira AR, Freitas Júnior IF. Riscos para o excesso de peso em adolescentes de diferentes classes econômicas. Rev Assoc Med Bras 2008;54:334-8. 